\title{
Modeling the glucose regulatory system in extreme preterm
}

\section{infants}

Aaron LE COMPTE ${ }^{1}, \mathrm{~J}_{\text {Geoffrey CHASE}}{ }^{2}$, Glynn RUSSELL ${ }^{3}$, Adrienne LYNN ${ }^{3}$, Chris HANN ${ }^{2}$, Geoffrey SHAW ${ }^{4}$, Xing-Wei WONG ${ }^{2}$, Amy BLAKEMORE ${ }^{1}$, Jessica LIN $^{2}$

1

Department of Mechanical Engineering, University of Canterbury

2

PhD, Department of Mechanical Engineering, University of Canterbury

3

MBChB, FRACP, Neonatal Department, Christchurch Women's Hospital

4

MbChB, FJFICM, Department of Intensive Care, Christchurch Hospital, Christchurch School of Medicine and Health Science, University of Otago

Work performed at:

- Neonatal Department, Christchurch Hospital

- Department of Mechanical Engineering, University of Canterbury

- Christchurch School of Medicine and Health Sciences, University of Otago

Address for Reprints: Reprints available from

Prof J. Geoffrey Chase,

Department of Mechanical Engineering

University of Canterbury,

Private Bag 4800

Christchurch

New Zealand

Email: geoff.chase@canterbury.ac.nz

Financial Support: New Zealand Tertiary Education Commission.

Keywords: Hyperglycemia, NICU, premature birth, Blood Glucose, Insulin 


\begin{abstract}
BACKGROUND

Premature infants represent a significant proportion of the neonatal intensive care population. Blood glucose homeostasis in this group is often disturbed by immaturity of endogenous regulatory systems and the stress of their condition. Hypo- and hyperglycemia are frequently reported in very low birth weight infants, and more mature infants often experience low levels of glycemia. A model capturing the unique fundamental dynamics of the neonatal glucose regulatory system could be used to develop better blood glucose control methods.
\end{abstract}

\title{
METHODS
}

A metabolic system model is adapted from adult critical care to the unique physiological case of the neonate. Integral-based fitting methods were used to identify time-varying insulin sensitivity and noninsulin mediated glucose uptake profiles. The clinically important predictive ability of the model was assessed by assuming insulin sensitivity was constant over prediction intervals of 1,2 and 4 hours forward and comparing model-simulated versus actual clinical glucose values for all recorded interventions. The clinical data included 1,091 glucose measurements over 3,567 total patient hours, along with all associated insulin and nutritional infusion data, for $\mathrm{N}=25$ total cases. Ethics approval was obtained from the Upper South A Regional Ethics Committee for this study.

\section{RESULTS}

The identified model had a median absolute percentage error of 2.4\% [IQR: $0.9 \%-4.8 \%$ ] between model-fitted and clinical glucose values. Median absolute prediction errors at 1, 2 and 4-hour intervals were 5.2\% [IQR: 2.5\%-10.3\%], 9.4\% [IQR: 4.5\%-18.4\%] and 13.6\% [IQR: 6.3\%-27.6\%] respectively.

\section{CONCLUSIONS}

The model accurately captures and predicts the fundamental dynamic behaviors of the neonatal metabolism well enough for effective clinical decision support in glycemic control. The adaptation from adult to a neonatal case is based on data from literature. Low prediction errors and very low fitting errors indicate that the fundamental dynamics of glucose metabolism in both premature neonates and critical care adults can be described by similar mathematical models. 


\section{Introduction}

Premature infants commonly demonstrate poor glycemic control. Physiologically, the neonate is in a transitional state from the complete dependence of the foetus to the complete independence of older infants and adults [1]. Hyperglycemia occurs in 40-80\% of very low birth weight infants in the neonatal intensive care unit (NICU) [1, 2]. The incidence of hyperglycemia increases with degree of prematurity and lower birth weight. Hypoglycemia is also a commonly reported perturbation in glucose homeostasis in both very low birth weight infants and more mature neonates $[3,4]$. An increasing body of literature links hyperglycemia to worsened outcomes in preterm neonates, but there have been no studies of sufficient power to demonstrate whether hyperglycemia itself is harmful, or is merely a reflection of disease severity. Associated morbidities include sepsis, increased ventilator dependence, retinopathy of prematurity, hospital length of stay, osmotic diuresis, electrolyte imbalance and intraventricular haemorrhage [5-11].

Conditions that cause stress to the infant increase the risk of hyperglycemia [12]. Increased secretion of counter-regulatory hormones due to the stress of their condition leads to a prominent rise in endogenously produced glucose and the rate of hepatic gluconeogenesis, as well as a reduction in insulin sensitivity. Nutritional support regimes with high carbohydrate content are also provided to increase neonate weight, but often compound the counter-regulatory response [10]. Additionally, lipids and lipid components in nutritional inputs, particularly free fatty acids, can promote hyperglycemia [13].

Hyperglycemic infants are often treated by glucose restriction [13-15]. However, prolonged severe glucose restriction may adversely affect the infant's nutritional status and may also be a reason for poor 
growth observed in these infants [14]. Hence, there is a need to balance the adverse effects of hyperglycemia with the need to provide enough nutrition to promote growth. As a result, there is an increasing use of insulin to help maintain both normal glucose levels and glucose uptake [16, 17].

Insulin is an anabolic hormone and promotes growth, while lowering glucose levels. However, endogenous deficiency or lack of effect due to high insulin resistance will have a negative impact on glycemic levels [14]. Additionally, studies have shown reduced proteolysis, and thus preservation of muscle mass associated with insulin therapy [18, 19], independent of glucose infusion [20]. However, in the neonate, immature insulin processing can also produce excessive pro-insulin, which is approximately 16x less effective than regular adult insulin, which may also explain why some poor control is exhibited in this population [21].

Tight blood glucose control in an adult intensive care population has been shown to reduce morbidity and mortality by up to $45 \%$ for patients with stays greater than 2 days [22-24]. Several recent studies have associated hyperglycemia with increased morbidity and mortality in neonatal cohorts [7-9]. Ertl et al [5] and Garg et al [6] linked hyperglycemia with increased risk of retinopathy of prematurity. Limited trials of glucose control using insulin in neonates have been reported [14, 18, 25-30], and insulin therapy did help to improve glucose tolerance in these limited trials.

Model based glycemic control methods using both insulin and/or nutrition modulation have been employed successfully in the control of hyperglycemia in adult critical care populations, as reviewed in Chase et al [31]. Model based methods allow information about the metabolic state of the patient, and infant in this case, to be inferred from serial blood glucose measurements and records of nutrition and insulin administration [32, 33]. Therefore, a model capturing the fundamental dynamics of the neonatal 
glucose regulatory system could be used to develop methods to better control blood glucose levels in this cohort, while also enhancing nutritional support and improving outcomes.

This paper presents the adaptation and validation of a metabolic system model for glucose control from adult critical care to the unique clinical and physiological case of the neonate. 


\section{Methods}

\section{Patient cohort}

Retrospective data from 25 episodes of insulin therapy treatment in the Christchurch Women's Neonatal Intensive Care Unit was used in the study. These infants were not part of any clinical trial, and were selected based on receiving insulin as a standard treatment for hyperglycemia, as insulin infusion data is a requirement to accurately produce model fits. All infants received IV insulin mixed with $0.9 \%$ saline administered using similar infusion sets, and insulin tubing was flushed with concentrated insulin solution prior to use to minimize adsorption. Ethics approval for the collection and publication of data was obtained from the Upper South A Regional Ethics Committee.

The clinical details of the cohort are shown in Table 1. The 25 episodes of insulin usage comprised 21 individual patients representing 3,567 hours of patient data. The data set used for model fitting contained 1,091 blood glucose measurements, and totals of insulin and dextrose administered every hour. Gestational age at birth ranged from 23 to 28.9 weeks, and birth weight ranged from 600 to 1,280 grams. Inclusion criteria were birth weight less than 1,500 grams and a period of treatment with insulin of at least 12 hours. Insulin was commenced when blood glucose concentration was persistently above $10 \mathrm{mmol} / \mathrm{L}$, at the discretion of the attending clinician, and insulin rate was titrated to blood glucose level. Dextrose infusion rates were determined by the attending clinician. The majority of blood glucose concentrations (74\%) were measured with the Bayer 850 blood gas analyzer (Bayer AG, Leverkusen, Germany), with the remaining measurements taken using the bedside GluoCard (Arkray, Inc., Kyoto, Japan) and i-Stat (Abbott Laboratories, Illinois, USA) devices. The reported coefficients of variation for glucose measurements for the blood gas analyzer, GlucoCard and i-Stat devices were 4.9\% [34], 2.4-6.3\%[35, 36], and 2.0-3.5\% [37] respectively. 
The system model used in this research is based on a clinically validated glucose regulatory system model for adult intensive care patients [32, 33]. The model has been modified to account for the main physiological differences based on available clinical data and kinetic studies in neonates. The overall model is defined:

$$
\begin{gathered}
\dot{G}(t)=-p_{G} \cdot G(t)-S_{I} \cdot G(t) \cdot \frac{Q(t)}{1+\alpha_{G} Q(t)}+\frac{P(t)+\left(P_{E N D} * m_{\text {body }}\right)-\left(C N S * m_{\text {brain }}\right)}{V_{G, \text { frac }}(t) * m_{\text {body }}} \\
\dot{Q}(t)=-k Q(t)+k I(t) \\
\dot{I}(t)=-\frac{n I(t)}{1+\alpha_{I} I(t)}+\frac{u_{e x}(t)}{\left(V_{I, \text { frac }} * m_{\text {body }}\right)}+e^{-\left(k_{I} \frac{u_{e x}(t)}{V_{i}}\right)} I_{B}
\end{gathered}
$$

Where $G(t)[\mathrm{mmol} / \mathrm{L}]$ is the total plasma glucose and $I(t)[\mathrm{mU} / \mathrm{L}]$ is the plasma insulin, exogenous insulin input is represented by $u_{e x}(t)$ [mU/min] and basal endogenous insulin secretion $I_{B}[\mathrm{mU} / \mathrm{L} / \mathrm{min}]$, with $k_{I}$ [L/mU.min] representing the suppression of basal insulin secretion in the presence of exogenous insulin. The effect of previously infused insulin being utilized over time is represented by $Q(t)$ [mU/L], with $k$ [1/min] accounting for the effective life of insulin in the system. Body weight and brain weight are denoted by $m_{\text {body }}[\mathrm{kg}]$ and $m_{\text {brain }}[\mathrm{kg}]$ respectively. Patient endogenous glucose clearance and insulin effect are $p_{G}[1 / \mathrm{min}]$ and $S_{I}[\mathrm{~L} /(\mathrm{mU} . \mathrm{min})]$, respectively. The parameter $V_{I, \text { frac }}[\mathrm{L} / \mathrm{kg}]$ is the insulin distribution volume per kilogram body weight and $n[1 / \mathrm{min}]$ is the constant first order decay rate for insulin from plasma. Total plasma glucose input is denoted $P(t)[\mathrm{mmol} / \mathrm{min}]$, endogenous glucose production is denoted by $P_{E N D}[\mathrm{mmol} / \mathrm{kg} / \mathrm{min}]$ and $V_{G, f r a c}(t)[\mathrm{L} / \mathrm{kg}]$ represents the glucose distribution volume per kilogram of body weight. CNS [mmol/kg/min] represents non-insulin mediated glucose uptake by the central nervous system. Michaelis-Menten functions are used to model saturation, with $\alpha_{I}$ [L/mU] used for the saturation of plasma insulin disappearance, and $\alpha_{G}[\mathrm{~L} / \mathrm{mU}]$ for the saturation of insulin-dependent glucose clearance. 


\section{Parameter values}

Table 2 summarizes the parameters used in this model with supporting clinical references. For this study, $k, n, \alpha_{I}, \alpha_{G}, C N S, I_{B}$ and $V_{I, f r a c}(t)$ are set to generic population values based on reported data. Prior clinical and model sensitivity studies with the similar adult model $[33,38]$ have shown this choice to be robust with respect to prediction of response to clinical interventions.

The distribution of fluids in the neonatal body is markedly different from children and adults. In particular, the extra-cellular fluid compartment is a much higher percentage of total body weight [3941]. It can also decrease over time as very preterm neonates can pass through a diuretic stage in the first few days of life [40,42], and this contraction of the extra-cellular fluid compartment may be a major contributor to weight loss in infants of this gestation and postnatal age [43]. Hence, a population-based, time-varying volume of glucose distribution as a proportion of body weight was created for the parameter $V_{G, \text { frac }}(t)$ based on the clinical data shown in Figure $1[43,44]$. The distribution volume for insulin $V_{I, f r a c}$ was set to a neonatal plasma volume of approximately $4.5 \%$ of the body weight expressed in kilograms [44, 45]. The most recently measured body weight was used in parameter value calculations, and was generally updated every 2 to 3 days.

The parameter CNS represents non-insulin mediated glucose uptake by the central nervous system. In contrast to the adult case, the brain represents a major source of glucose uptake in infants, due to their larger brain-to-body weight ratio [20, 46]. Glucose metabolism by the brain is relatively constant [47] and independent of insulin concentration [1]. The value used for CNS in this study was $8.8 \mu \mathrm{mol} / \mathrm{min} / 100 \mathrm{~g}$ brain weight $(0.088 \mathrm{mmol} / \mathrm{min} / \mathrm{kg}$ brain weight $)$ based on [48], where the average brain weight of the preterm infant at the age used in this cohort was estimated at $14 \%$ of body weight [49]. 
With respect to saturation effects, Farrag et al [46] found no plateau in the rate of glucose uptake at several increasing levels of insulin infusion during clamp tests. This result suggests that insulin mediated glucose uptake does not saturate at the insulin delivery rates typically encountered in neonatal intensive care. Therefore, the parameter representing saturation of insulin mediated glucose uptake, $\alpha_{G}$, was set to zero in this study. This lack of saturation effect is significantly different to the adult case, where the significant glucose uptake saturation can occur [38, 50-52].

The parameter $n$ represents the disappearance of insulin from plasma. Factors influencing this parameter include transport into the interstitial space and removal by the liver and kidneys. Farrag, et al identified that the rate of insulin clearance was much higher in neonates compared to a group of healthy adults [46]. In this study, a value of $n=0.9$ was used, compared to 0.16 in adults [33], as derived from neonatal insulin infusion studies [18, 19, 46, 53].

The parameter $I_{B}$ represents basal insulin secretion in the presence of no exogenous insulin input. This parameter provides an estimate of basal insulin secretion to better model the actual physiological situation. It also makes the model robust to the sometimes frequent periods where no exogenous insulin is administered. This parameter was set to a population constant of $10 \mathrm{mU} / \mathrm{L} / \mathrm{min}$ to achieve steady state basal insulin concentrations similar to reported clinical results [46, 53]. It is assumed that exogenous insulin administration suppresses endogenous insulin secretion and the parameter $k_{I}$ broadly captures this suppression effect. Published clinical data was not available to quantify the effective life of insulin $(k)$ and plasma insulin disappearance saturation parameters $\left(\alpha_{I}\right)$, and it was therefore assumed that these values do not significantly differ from adults [33]. It should also be noted that a constant excretion would be expected in the presence of relatively constant nutritional infusions. The resulting plasma insulin model was compared to reported plasma insulin concentrations from constant insulin infusion studies in neonates [18, 19, 46, 53, 54]. 
The parameter $P_{E N D}$ represents endogenous glucose production, which can be measured in tracer studies [20, 53, 55-59]. Endogenous glucose production is decreased in the presence of increased blood glucose concentration and increased plasma insulin concentration [59, 60]. Because of the fragile nature of this population the true fasting rate of glucose production cannot be determined. In addition, almost all such studies are performed concurrently with parenteral nutrition to avoid underfeeding the low birth weight neonate. Figure 2 plots the average endogenous glucose production against blood glucose concentration for the available literature reporting tracer studies in preterm neonates [20, 53, 55-59]. At $G=0$, the $p_{G}$ and $S_{I}$ terms in Equation 1 become zero. Thus, the range of values for $P_{E N D}=$ [0.0172 to $0.0312 \mathrm{mmol} / \mathrm{kg} / \mathrm{min}$ ] suitable for this model formulation were obtained from Figure 2 at $G=0$ (the intercept with the y-axis). Bootstrapping with replacement ( $\mathrm{N}=1000)$ was used to determine the mean intercept and the upper and lower $5 \%$ of possible intercepts that define this range.

\section{Parameter identification and sensitivity analysis}

The parameters $P_{E N D}, p_{G}$ and $S_{I}$ are the remaining parameters to explicitly determine from the data. As a result of using $P_{E N D}$ at $G=0$, the parameters $p_{G}$ and $S_{I}$ incorporate the effects of insulin and blood glucose concentration to inhibit endogenous glucose production. Finally, the range of $P_{E N D}$ in Figure 2 delimits the acceptable values for this parameter, but not a specific population of patient-specific value.

A grid search was performed to determine the optimal population values of $P_{E N D}$ and $p_{G}$ for this cohort. In particular, $p_{G}$ was found to be relatively constant in an adult intensive care population [33], and is initially assumed so here.

To this end, the time-varying parameter $S_{I}$ was fitted to the clinical data using several values of $P_{E N D}$ held constant within the range of $[0.01442-0.03396] \mathrm{mmol} / \mathrm{kg} / \mathrm{min}$ based on Figure 2 . The range of 
$P_{E N D}$ assessed in simulation extends beyond the 5\%-95\% range shown in Figure 2 to more fully observe the effect of this model parameter. The value of $p_{G}$ was also held constant within the range [0.0005-0.02] $\mathrm{min}^{-1}$. This range of $p_{G}$ is based on a range of adult results with no further data available for the neonate.

The insulin effect parameter, $S_{I}$, is allowed to vary every hour. The integral-based fitting method of Hann et al [33] was employed for identifying $S_{I}$ over time using retrospective blood glucose concentrations $(G)$, insulin administration $\left(u_{e x}(t)\right)$ and nutritional infusion rates $(P(t))$. The resulting time-varying $S_{I}$ profile smoothed using a 3-point moving average to reduce the effects of sensor noise. The smoothed $S_{I}$ profile was then used to generate a model derived BG curve to compare to the clinical data and thus assess the model. A total of 2,000 such assessments were made over the ranges of $p_{G}$ and $P_{E N D}$ for the 25 patient cohort with $S_{I}$ identified for each $\left(p_{G}, P_{E N D}\right)$ set of values. The optimal $P_{G}$ and $P_{E N D}$ values are presented in the Results and Table 2.

The population constants $\alpha_{G}, \alpha_{I}, C N S, k, n, V_{G}$ and $V_{I}$ were then varied by $-50 \%,-10 \%$, $+10 \%$ and $+50 \%$ to address model sensitivity to a particular patient constant and the appropriateness of using a particular population-based constant. The retrospective cohort data was re-fitted for each parameter variation and model performance assessed using BG fit and prediction analyses.

The median and IQR of absolute fitting error and prediction error were the metrics used to compare the ability of the model to fit the data for any set of parameters. Fitting error was assessed by analyzing the absolute percentage difference between measured blood glucose concentration and identified model generated blood glucose concentration. Prediction error is clinically more important and was assessed over 1, 2, 3 and 4-hour intervals. Predictions were generated at each blood glucose measurement by holding all parameter values, in particular $S_{I}$, constant over the prediction interval and performing a 
forward simulation of Equations (1)-(3). Only data up to that time point are used in forward predictions. This method reflects the use of this model in adult critical care trials. Prediction error was analyzed by comparing the error between the predicted blood glucose concentration and its measured value, or where a measurement was not available the prediction was compared to a linearly interpolated value between two appropriate blood glucose measurements. The optimal or best $\left(p_{G}, P_{E N D}\right)$ values are thus those that provide the best outcomes in these fitting and prediction metrics. 


\section{Results}

\section{Plasma insulin model}

Figure 3 shows the plasma insulin model and population constants are valid for a wide range of reported insulin concentrations from steady exogenous insulin infusions. Typical insulin infusion rates expected in neonatal control scenarios would occupy the left half of the range shown in Figure 3, below $0.3 \mathrm{U} / \mathrm{kg} / \mathrm{hr}$. Agus and colleagues $[10,11]$ used supra-physiological concentrations of insulin to determine the effects of insulin on protein breakdown. Thus, they provide further data points but at clinically irrelevant values.

Typical steady state insulin concentrations for the adult insulin model for similar weight-based rates of insulin infusion are shown in Figure 3. Neonatal plasma insulin concentrations are significantly lower than adults for similar weight-adjusted rates of exogenous insulin infusion [39]. This result may represent a much higher metabolic clearance rate of insulin in the neonate. To date, the specific mechanisms that create a higher metabolic clearance rate in neonates remain as open questions. The goal of this study was to generate a model designed for control purposes that matches observable insulin kinetics in the neonate and further investigation of physiological mechanisms of insulin transport is outside the scope of this research.

\section{Parameter and sensitivity analysis}

Figure 4 shows that fit error plateaus at minimum levels for values of $p_{G} \leq 0.01 \mathrm{~min}^{-1}$ and $P_{E N D} \geq 0.02$ $\mathrm{mmol} / \mathrm{kg} / \mathrm{min}$. These results suggests that a range of values for $p_{G}$ and $P_{E N D}$ will provide robust fitting and prediction performance. Figure 4 also shows the 1, 2, 3 and 4-hour prediction errors over these

same ranges result in similar plateaus of minimal error for $p_{G}<0.01 \mathrm{~min}^{-1}$ and $P_{E N D}>0.02$ $\mathrm{mmol} / \mathrm{kg} / \mathrm{min}$. 
The sum of the maximum five BG fitting errors per patient profile was used to choose the optimum values of $p_{G}$ and $P_{E N D}$. The combination of $p_{G}=0.003 \mathrm{~min}^{-1}$ and $P_{E N D}=0.02838 \mathrm{mmol} / \mathrm{kg} / \mathrm{min}$ provided the smallest sum of maximum fitting errors across the entire cohort. These final values are also shown in the lower part of Table 2. Thus, an example of the resulting model for a 750 gram infant born at 28 weeks gestational age, receiving an insulin infusion of $0.05 \mathrm{U} / \mathrm{kg} / \mathrm{hr}$ and a dextrose infusion of $8 \mathrm{mg} / \mathrm{kg} / \mathrm{min}$ may be found by inserting the value of Table 2 into Equations (1)-(3) to yield Equations (4)-(6). The system of equations may be solved and the $S_{I}$ parameter fitted using the integralbased fitting method of Hann et al [33].

$$
\begin{gathered}
\dot{G}(t)=-0.003\left[\mathrm{~min}^{-1}\right] \cdot G(t)-S_{I} \cdot G(t) \cdot Q(t) \\
+\frac{0.033[\mathrm{mmol} / \mathrm{min}]+(0.02838[\mathrm{mmol} / \mathrm{kg} / \mathrm{min}] \times 0.75[\mathrm{~kg}])-0.00924[\mathrm{mmol} / \mathrm{min}]}{0.56[\mathrm{~L} / \mathrm{kg}] \times 0.75[\mathrm{~kg}]} \\
\dot{Q}(t)=-0.0086\left[\mathrm{~min}^{-1}\right] Q(t)+0.0086\left[\mathrm{~min}^{-1}\right] I(t) \\
\dot{I}(t)=-\frac{0.90\left[\mathrm{~min}^{-1}\right] . I(t)}{1+1.70 \times 10^{-3}\left[\mathrm{~min}^{-1}\right] \cdot I(t)}+\frac{0.625[\mathrm{mU} / \mathrm{min}]}{0.045[\mathrm{~L} / \mathrm{kg}] \times 0.75[\mathrm{~kg}]} \\
+e^{-\left(0.1[L /(\mathrm{mU} \cdot \mathrm{min})] \frac{0.625[\mathrm{mU} / \mathrm{min}]}{0.045[\mathrm{~L} / \mathrm{kg}] \times 0.75[\mathrm{~kg}]}\right)} \times 10[\mathrm{mU} / \mathrm{L} / \mathrm{min}]
\end{gathered}
$$

The results of the population constant sensitivity analysis shown in Figure 5 indicate that model performance is relatively insensitive to the variations in population constants introduced here. The volume of glucose distribution, $V_{G}$, created the greatest effect on model performance at $-50 \%$ of the population value used here, which is a very large change for a typically well known or understood parameter. A reduction of $V_{G}$ of $50 \%$ brings this volume closer to the value used for adults in proportion to body weight. Thus, recognizing the increased proportion of body weight taken up as extra-cellular fluid in neonates is seen to be relevant to accurate glucose control. This last result provides a further model validation, in that good model performance requires accurate neonatal physiology where such differences to adults are known to exist. 


\section{Model performance}

The median cohort model fit error was $2.4 \%$ [IQR: $0.9 \%-4.8 \%$ ] or $0.2 \mathrm{mmol} / \mathrm{L}$ [IQR: $0.1-0.4$ $\mathrm{mmol} / \mathrm{L}$ ] for the 3,567 hours of patient data. The smoothing process attempts to remove nonphysiological sources of noise such as errors in BG measurements and recorded insulin/dextrose inputs, and results in a more physiological $S_{I}$ parameter profile at the expense of a potentially greater fit error. A sample parameter identifications and model-generated BG fit is presented in Figure 6.

Table 3 shows the median fitting error for all patients and the retrospective lognormal blood glucose mean, multiplicative lognormal standard deviation, median insulin use and median dextrose delivered. The results show a relatively constant level of fitting error spanning cases of relatively unstable blood glucose, indicated by a higher standard deviation and/or higher mean blood glucose. They also show varying levels of insulin and glucose administration over these same results. Thus, the model can be seen to account for a range of different patient responses, which include a range of $S_{I}$ parameter values indicating these patients can be highly dynamic metabolically.

Table 4 shows the median absolute percentage prediction error for 1, 2, 3 and 4-hour prediction intervals. Results are compared to blood glucose measurements or linearly interpolated between retrospective measurements where none are available for a given interval. The cohort median prediction error was 5.2\% at 1 hour, $9.4 \%$ at 2 hours, $11.9 \%$ at 3 hours and $13.6 \%$ at 4 hours. Blood glucose prediction errors did not change over time for each patient. This result also emphasizes the importance of frequent blood glucose measurement to provide tighter continuous control. Figure 7 shows the distribution of absolute blood glucose prediction error, with increasing variance as the prediction interval increases. 
Figure 8 shows part of the record for Patient 13. This episode is dominated by the BG measurement of $31.2 \mathrm{mmol} / \mathrm{L}$ at 7 hours. The gradual decrease in BG over the next 11 hours suggests this measurement was not due to errors in the measuring device. This patient had severe metabolic imbalances, was relatively clinically unstable and did not survive ICU. A glucose/insulin mixture was administered at 6 hours that contained $0.26 \mathrm{U}$ insulin in $8 \mathrm{~mL} / \mathrm{kg}$ of $50 \%$ dextrose. Interestingly, the model was able to account for this relatively extreme deviation in blood glucose concentration, and accurately captured the impact of this sudden large amount of added dextrose on BG concentration.

Figure 9 shows the cumulative distribution function of the identified time-varying $S_{I}$ profile for each patient, with median and 5\%-95\% range overlaid. Each patient's insulin sensitivity profile is unique, yet there are no significantly outlying patient profiles. Figure 10 compares histograms of the empirical cumulative distribution functions of model-fitted $S_{I}$ for the neonatal cohort of this paper to 393 adult intensive care patients totaling 44,386 hours of fitted data [16]. The median $S_{I}$ for neonates was $0.68 \times 10^{-3} \mathrm{~L} /(\mathrm{mU} \cdot \mathrm{min})$, compared to $0.24 \times 10^{-3} \mathrm{~L} /(\mathrm{mU} \cdot \mathrm{min})$ for adults, and the $5 \%-95 \%$ data interval was $[0.17-1.70] \times 10^{-3} \mathrm{~L} /(\mathrm{mU} . \mathrm{min})$ for neonates and $[0.06-0.79] \times 10^{-3} \mathrm{~L} /(\mathrm{mU} . \mathrm{min})$ for adults respectively.

Finally, Figure 11 presents the model generated fit for Patient 23 and highlights a sustained period of low $S_{I}$ and large swings in blood glucose concentration. This period of unstable glycemia contrasts with a more typical patient blood glucose time-course presented in Figure 6. 


\section{Discussion}

Tight glycemic control without increased risk of hypoglycemia has proven difficult to achieve in intensive care in both adults [61] and neonates [62]. Moderately elevated blood glucose levels are recently more tolerated or recommended [63] because of the fear of hypoglycemia and higher nursing effort frequently associated with TGC [64]. A recent study by Alsweiler and colleagues [65] found that while all surveyed neonatal units would consider use of insulin for hyperglycemia, the threshold for commencing insulin infusions varied, as well as the choice of glycemic management using altered dextrose infusion rates. Most units reported little to no hypoglycemia, and these findings agreed with previous surveys that found a lack of consensus on the management of neonatal hyperglycemia, possibly reflecting the lack of data available in this area [65]. Most studies of hyperglycemia and outcomes in neonates are retrospective, and it is difficult to ascertain whether high glucose levels are a cause or a symptom of conditions such as sepsis [8]. Maintenance of normoglycemia with exogenous insulin has been found to improve innate immunity and reduce catabolism, acidosis and inflammation in rabbit models of critical illness [66], which may or may not hold in the neonatal case. More prospective studies are thus required to elucidate the link between tight glucose control and neonatal outcomes. Model-based control may be useful in exploring these relationships by providing targeted glucose control whilst concurrently minimizing the risk of hypoglycemia.

Insulin sensitivity can be measured in-vivo using the euglycemic hyperinsulinemic clamp test [60], where a euglycemic concentration of glucose is maintained at several different levels of insulinemia. This clinically intensive process also requires several blood samples and is thus difficult to perform in clinically fragile low birth weight infants who also possess a relatively very small blood volume for this procedure. Three euglycemic hyperinsulinemic clamps that incorporated glucose tracer measurements 
on preterm infants were available in the literature [46, 53, 67]. An additional study [45] used a modified minimal model analysis to generate an insulin sensitivity index. Results in all cases showed a significant spread of insulin sensitivities for otherwise similar infants, similar to what is seen in this study.

Figure 9 shows location and spread of the $S_{I}$ parameter for this NICU cohort and Figure 10 presents a comparison of the location and spread of $S_{I}$ for NICU and adult ICU patients. The range and variation of model-fitted $S_{I}$ has been studied in adult critical care populations using similar models [68, 69]. However, this is the first time this form of modeling has been applied to a neonatal cohort. Two reasons for this higher median $S_{I}$ in neonates are higher rates of glucose turnover and higher metabolic clearance of insulin resulting in lower plasma insulin concentrations. More specifically, Farrag et al found greater peripheral sensitivity to insulin compared to adult controls [46]. Hertz et al [20] found their estimate of non-insulin mediated glucose uptake to be similar to that of normal adults. Thus, the lower insulin concentrations and greater peripheral sensitivity to insulin in neonates suggests higher numerical values of $S_{I}$ compared to adults. However, this finding may also reflect differences in endogenous glucose and insulin production between the two cohorts.

More specifically, Figure 9 shows that each patient has a uniquely identified $S_{I}$ parameter profile. The intra-patient variability of response to exogenous insulin is significant in the neonate, increasing the importance of accurate identification for control. Thus, noting the wider spread in Figure 10, NICU patients exhibit high inter-patient variation relative to adults. Thus, such differences must be accounted for when designing glucose control protocols for neonates, or adapting successful protocols from the adult critical care case. An adaptive control scheme must be able to dose insulin appropriately to the unique identified patient state. As a result, it can be seen that such an adaptive method will be required to provide tight control for all patients, rather than fixed clinical protocols. 
The $P_{E N D}$ term represents the regulatory response of the body to endogenously produce glucose to avoid hypoglycemia. To measure the true rate of endogenous glucose production, the neonate would be required to undergo a fast for several hours, which is generally not possible for clinical and ethical reasons [15]. The estimates of basal endogenous glucose production derived in this study are higher than the values seen in adults $[20,70]$. The explanation for this difference is that the extra glucose is required in infants for growth and to supply the glucose requirements of the relatively large brain in the infant compared to total body weight [71].

In particular, it has been shown that endogenous glucose production (via glycogenolysis and gluconeogenesis, primarily from the liver) may not be suppressed in the preterm infant by exogenous glucose infusions [46, 55]. Specifically, Farrag et al [46] found a maximal suppression of endogenous glucose production of $58 \%$ of basal rates. This result is in marked contrast to the healthy adult who can effectively completely suppresses endogenous glucose production [46]. However, it is similar to the critically ill adult $[31,72]$. In this paper, some of this effect is captured by the parameters $p_{G}$ and $S_{I}$, which incorporate contributions of increased insulin concentration [20] and plasma glucose concentration [15] to decreasing net hepatic glucose output.

Several studies have reported that many neonates produce an inadequate glucose raising response to decreases in glucose infusion [58] and to a bolus of glucagon [73]. Additionally, it has been shown that many preterm infants have concentrations of hepatic glucose-6-phosphatase, the rate limiting enzyme in gluconeogenesis, below the limit of normal term infants [74]. Glycogen stores in premature infants to produce further glucose for use are also relatively sparse [16, 74]. Low birth weight infants therefore must be fed adequately to prevent long-term deficiencies in growth and development [75]. However, initiation of feeding has also been linked to hyperglycemia [10, 13]. Thus, control of hyperglycemia 
whilst maintaining an adequate nutrition rate may be one factor of benefit for neonatal growth and development.

Hence, the goal of this model is to provide a vehicle for real-time blood glucose control. It is therefore desirable to minimize the number of patient-specific parameters to be identified to avoid identifiability issues with limited (glucose only) measurements. This issue is particularly important in this neonatal case where real-time tracer studies are not viable and control must be based upon a very minimal number and volume of blood samples compared to the adult case. This latter point is a salient difference from the critically ill adult case where the blood sampling frequency used is primarily a question of clinical burden. Therefore, the model must provide better longer term prediction performance at 2 or more hours forward, which is dependent on good identification.

The predictive ability presented is similar to other studies that have successfully applied similar models to control glycemia in adult intensive care patients [33]. The model sufficiently captures the varied dynamics of the neonatal cohort. Model prediction error was independent of length of accumulated data for each patient. This result possibly reflects that hour-to-hour changes in response to insulin are largely random, similar to variations in critical care adults [68, 69]. The parameter sensitivity analysis revealed the population parameter that appeared to have the most influence on model fit and prediction errors was the glucose volume of distribution, $V_{G}$. Outside this parameter, the low sensitivity of model performance to the remaining parameters is similar to the adult case [33]. This result may imply that, with the right neonatal parameters, models of similar structure give similar fit and prediction performance in adults and neonates, which may be unexpected given the differing external clinical management of metabolism in these patient groups. 
It should be noted that the frequency of measurement of retrospective data can influence the accuracy of blood glucose predictions, where some dynamics may be missed by sparse data collection. This issue is especially evident in the relatively poor prediction results for Patient 23 . Figure 11 shows a part of the record for Patient 23, highlighting a period of rapid change in blood glucose. This patient experienced much more rapid swings in blood glucose compared to the rest of the cohort. This period of glycemic instability coincided with steroid and antibiotic administration, very unstable respiratory periods requiring resuscitation, a number of new IV lines, and some BG measurements taken using a GlucoCard glucometer, rather than the typical blood gas analyzer. In general, more frequent blood glucose measurements would enable the model to more accurately capture such periods of rapid change in glucose concentration, as well as provide insight to the contribution to unstable glucose due to patient condition and noise from measurement devices.

Published data was not available to directly estimate the insulin removal saturation parameter $\alpha_{I}$, so this parameter was held at the same value as the adult case. Figure 3 shows that the effect of $\alpha_{I}$ is only relevant at very high insulin infusion rates, and that the value employed provides a good fit to the supra-physiological results of $[18,19]$. The insulin model in adults exhibits instability for insulin infusion rates greater than approximately $18 \mathrm{U} / \mathrm{hr}$ for a typical 70kg adult due to the insulin removal saturation term $\alpha_{I}$. The adult model was designed for use for insulin in the range of 0 - $8 \mathrm{U} / \mathrm{hr}(0-0.11$ $\mathrm{U} / \mathrm{kg} / \mathrm{hr}$ ). The dynamics of the neonatal model allow stability for a insulin infusion rates up to $1.4 \mathrm{U} / \mathrm{hr}$ for a typical $1 \mathrm{~kg}$ infant, and represents an infusion rate approximately $3 \mathrm{x}-9 \mathrm{x}$ higher than would be used for control.

The major assumption used in the predictions made in this study is that the in $S_{I}$ parameter is constant over the prediction interval. Hence, longer intervals will subsequently show potentially greater variation as patient condition has a longer time and thus more opportunity to evolve. However, Figure 8 
shows the mode of the error distribution remains relatively constant at approximately $4-7 \%$ over all four 1-4 hour prediction intervals. Thus, the increasing likelihood of changes over time in $S_{I}$ are evident, as seen previously in the adult population $[33,68,76]$. Stochastic modeling of the $S_{I}$ parameter [77], as applied in adult critical care patients [68], could provide a more accurate forecast of future $S_{I}$ values and thus create confidence intervals for predicted blood glucose concentrations that account for this variation and would thus provide better clinical guidance. 


\section{Conclusions}

A model of the glucose regulatory system has been adapted from adult critical care to the neonatal case. Model fits of the $S_{I}$ parameter were generated for 25 patient records and 3,567 hours of retrospective data. Adaptation of several physiological model parameters, including insulin clearance rate, endogenous production rate and volumes of distribution, resulted in an average fitting error of $2.4 \%$. The predictive ability of the model was assessed by assuming parameters were constant over the interval. Prediction error at 1, 2 and 4-hour intervals were 5.2\%, 9.4\% and 13.6\% respectively. The model performance is within variations that would also account for dynamic patient evolution. The model thus provides a first in-silico result for modeling the metabolic dynamics of the low-birth weight preterm infant. It also creates a platform towards better metabolic clinical management of glycemia in neonates. 


\section{Conflict of interest statement}

The authors state they have no outstanding conflicts of interest.

\section{Financial Support}

New Zealand Tertiary Education Commission. This funding source had no role in the study design, collection, analysis or interpretation of the data, in the writing of the manuscript or the decision to submit the manuscript. 


\section{References}

[1] R. M. Cowett and H. M. Farrag, "Selected principles of perinatal-neonatal glucose metabolism," Semin Neonatol, vol. 9, pp. 37-47, Feb 2004.

[2] H. S. Dweck and G. Cassady, "Glucose Intolerance in Infants of Very Low Birth Weight," Pediatrics, vol. 53, p. 189, 1974.

[3] C. B. Duvanel, et al., "Long-term effects of neonatal hypoglycemia on brain growth and psychomotor development in small-for-gestational-age preterm infants," J Pediatr, vol. 134, pp. 492-8, Apr 1999.

[4] L. O. Lubchenco and H. Bard, "Incidence of hypoglycemia in newborn infants classified by birth weight and gestational age," Pediatrics, vol. 47, pp. 831-8, May 1971.

[5] T. Ertl, et al., "Relationship between hyperglycemia and retinopathy of prematurity in very low birth weight infants," Biology of the neonate, vol. 89, pp. 56-9, 2006.

[6] R. Garg, et al., "Hyperglycemia and retinopathy of prematurity in very low birth weight infants," Journal of perinatology, vol. 23, pp. 186-94, Apr-May 2003.

[7] S. P. Hays, et al., "Hyperglycemia Is a Risk Factor for Early Death and Morbidity in Extremely Low Birth-Weight Infants," Pediatrics, vol. 118, pp. 1811-1818, 2006.

[8] L. S. Kao, et al., "Hyperglycemia and morbidity and mortality in extremely low birth weight infants," Journal of perinatology, vol. 26, pp. 730-6, Dec 2006.

[9] N. J. Hall, et al., "Hyperglycemia is associated with increased morbidity and mortality rates in neonates with necrotizing enterocolitis," Journal of pediatric surgery, vol. 39, pp. 898-901; discussion 898-901, Jun 2004.

[10] D. I. Alaedeen, et al., "Total parenteral nutrition-associated hyperglycemia correlates with prolonged mechanical ventilation and hospital stay in septic infants," J Pediatr Surg, vol. 41, pp. 239-44; discussion 239-44, Jan 2006.

[11] K. Heimann, et al., "Are recurrent hyperglycemic episodes and median blood glucose level a prognostic factor for increased morbidity and mortality in premature infants </=1500 g?," Journal of perinatal medicine, vol. 35, pp. 245-8, 2007.

[12] G. R. Ditzenberger, et al., "Continuous insulin intravenous infusion therapy for VLBW infants," J Perinat Neonatal Nurs, vol. 13, pp. 70-82, Dec 1999.

[13] A. H. Hemachandra and R. M. Cowett, "Neonatal Hyperglycemia," Pediatrics in Review, vol. 20, pp. 16e-24, 1999/7/1 1999.

[14] F. Thabet, et al., "Continuous insulin infusion in hyperglycemic very-low-birth-weight infants receiving parenteral nutrition," Clin Nutr, vol. 22, pp. 545-7, Dec 2003.

[15] H. M. Farrag and R. M. Cowett, "Glucose homeostasis in the micropremie," Clinics in Perinatology, vol. 27, pp. 1-22, 2000.

[16] P. Mena, et al., "Insulin homeostasis in the extremely low birth weight infant," Semin Perinatol, vol. 25, pp. 436-46, Dec 2001.

[17] V. Kairamkonda, "Does continuous insulin infusion improve glycemic control and nutrition in hyperglycemic very low birth weight infants?," Arch Dis Child, vol. 91, pp. 76-79, 2006/1/1 2006.

[18] M. S. Agus, et al., "Intravenous insulin decreases protein breakdown in infants on extracorporeal membrane oxygenation," Journal of pediatric surgery, vol. 39, pp. 839-44; discussion 839-44, Jun 2004.

[19] M. S. Agus, et al., "The Effect of Insulin Infusion Upon Protein Metabolism in Neonates on Extracorporeal Life Support," Annals of Surgery Scientific Papers of the 126th Annual Meeting of the American Surgical Association, vol. 244, pp. 536-544, 2006. 
[20] D. E. Hertz, et al., "Intravenous glucose suppresses glucose production but not proteolysis in extremely premature newborns," The Journal of clinical investigation, vol. 92, pp. 1752-8, Oct 1993.

[21] D. Mitanchez-Mokhtari, et al., "Both Relative Insulin Resistance and Defective Islet \{beta $\}$ Cell Processing of Proinsulin Are Responsible for Transient Hyperglycemia in Extremely Preterm Infants," Pediatrics, vol. 113, pp. 537-541, 2004/3/1 2004.

[22] G. Van den Berghe, et al., "Intensive insulin therapy in the critically ill patients," $N$ Engl J Med, vol. 345, pp. 1359-1367, Nov 82001.

[23] J. S. Krinsley, "Decreased mortality of critically ill patients with the use of an intensive glycemic management protocol," Crit Care Med, vol. 31, p. A19, 2003.

[24] J. G. Chase, et al., "Implementation and evaluation of the SPRINT protocol for tight glycemic control in critically ill patients: a clinical practice change," Critical Care, vol. 12, p. R49, 2008.

[25] J. W. Collins, Jr., et al., "A controlled trial of insulin infusion and parenteral nutrition in extremely low birth weight infants with glucose intolerance," The Journal of pediatrics, vol. 118, pp. 921-7, Jun 1991.

[26] Y. E. Vaucher, et al., "Continuous insulin infusion in hyperglycemic, very low birth weight infants," Journal of pediatric gastroenterology and nutrition, vol. 1, pp. 211-7, 1982.

[27] N. D. Binder, et al., "Insulin infusion with parenteral nutrition in extremely low birth weight infants with hyperglycemia," The Journal of pediatrics, vol. 114, pp. 273-80, Feb 1989.

[28] S. M. Ng, et al., "Continuous insulin infusion in hyperglycemic extremely-low- birth-weight neonates," Biol Neonate, vol. 87, pp. 269-72, 2005.

[29] S. Ostertag, et al., "Insulin pump therapy in the very low birth weight infant," Pediatrics, vol. 78, pp. 625-630, 1986/10/1 1986.

[30] K. S. Kanarek, et al., "Continuous infusion of insulin in hyperglycemic low-birth weight infants receiving parenteral nutrition with and without lipid emulsion," J Parenter Enteral Nutr, vol. 15, pp. 417-20, Jul-Aug 1991.

[31] J. Chase, et al., "Model-based glycemic control in critical care - a review of the state of the possible," Biomedical Signal Processing and Control, vol. 1, pp. 3-21, 2006.

[32] J. G. Chase, et al., "Model-based insulin and nutrition administration for tight glycemic control in critical care," Curr Drug Deliv, vol. 4, pp. 283-96, Oct 2007.

[33] C. E. Hann, et al., "Integral-based parameter identification for long-term dynamic verification of a glucose-insulin system model," Comput Methods Programs Biomed, vol. 77, pp. 259-270, Mar 2005.

[34] A. C. Peet, et al., "Near-patient testing of blood glucose using the Bayer Rapidlab 860 analyser in a regional neonatal unit," Ann Clin Biochem, vol. 39, pp. 502-8, Sep 2002.

[35] S. Arens, et al., "Evaluation of Glucocard Memory 2 and Accutrend sensor blood glucose meters," Clin Chem Lab Med, vol. 36, pp. 47-52, Jan 1998.

[36] K. Devreese and G. Leroux-Roels, "Laboratory assessment of five glucose meters designed for self-monitoring of blood glucose concentration," Eur J Clin Chem Clin Biochem, vol. 31, pp. 829-37, Dec 1993.

[37] T. Mock, et al., "Evaluation of the i-STAT system: a portable chemistry analyzer for the measurement of sodium, potassium, chloride, urea, glucose, and hematocrit," Clin Biochem, vol. 28, pp. 187-92, Apr 1995.

[38] X. W. Wong, et al., "Model predictive glycemic regulation in critical illness using insulin and nutrition input: a pilot study," Med Eng Phys, vol. 28, pp. 665-81, Sep 2006.

[39] J. Simpson and T. Stephenson, "Regulation of extracellular fluid volume in neonates," Early Hum Dev, vol. 34, pp. 179-90, Oct 1993.

[40] J. M. Lorenz, et al., "Phases of fluid and electrolyte homeostasis in the extremely low birth weight infant," Pediatrics, vol. 96, pp. 484-9, Sep 1995. 
[41] G. Hartnoll, et al., "Body water content of extremely preterm infants at birth," Archives of disease in childhood, vol. 83, pp. F56-9, Jul 2000.

[42] K. S. Bidiwala, et al., "Renal function correlates of postnatal diuresis in preterm infants," Pediatrics, vol. 82, pp. 50-8, Jul 1988.

[43] G. B. Avery, et al., Neonatology : pathophysiology and management of the newborn., 4th ed.: Philadelphia : Lippincott, c1994., 1994.

[44] G. Cassady, "Plasma volume studies in low birth weight infants," Pediatrics, vol. 38, pp. 10207, Dec 1966.

[45] J. A. Leipala, et al., "Blood volume assessment with hemoglobin subtype analysis in preterm infants," Biol Neonate, vol. 84, pp. 41-4, 2003.

[46] H. M. Farrag, et al., "Persistent glucose production and greater peripheral sensitivity to insulin in the neonate vs. the adult," Am J Physiol, vol. 272, pp. E86-93, Jan 1997.

[47] R. Gruetter, et al., "Steady-state cerebral glucose concentrations and transport in the human brain," J Neurochem, vol. 70, pp. 397-408, Jan 1998.

[48] W. J. Powers, et al., "Cerebral glucose transport and metabolism in preterm human infants," $J$ Cereb Blood Flow Metab, vol. 18, pp. 632-8, Jun 1998.

[49] K. C. Ho, et al., "Newborn brain weight in relation to maturity, sex, and race," Annals of neurology, vol. 10, pp. 243-6, Sep 1981.

[50] R. L. Prigeon, et al., "The effect of insulin dose on the measurement of insulin sensitivity by the minimal model technique. Evidence for saturable insulin transport in humans," J Clin Invest, vol. 97, pp. 501-7, Jan 151996.

[51] J. G. Chase, et al., "Impact of insulin-stimulated glucose removal saturation on dynamic modelling and control of hyperglycemia," International Journal of Intelligent Systems Technologies and Applications (IJISTA), vol. 1, pp. 79-94, 2004.

[52] A. Natali, et al., "Dose-response characteristics of insulin action on glucose metabolism: a nonsteady-state approach," Am J Physiol Endocrinol Metab, vol. 278, pp. E794-801, May 2000.

[53] B. B. Poindexter, et al., "Exogenous insulin reduces proteolysis and protein synthesis in extremely low birth weight infants," The Journal of pediatrics, vol. 132, pp. 948-53, Jun 1998.

[54] K. Beardsall, et al., "Early elective insulin therapy can reduce hyperglycemia and increase insulin-like growth factor-I levels in very low birth weight infants," The Journal of pediatrics, vol. 151, pp. 611-7, 617 e1, Dec 2007.

[55] R. M. Cowett, et al., "Persistent glucose production during glucose infusion in the neonate," $J$ Clin Invest, vol. 71, pp. 467-75, Mar 1983.

[56] A. Sunehag, et al., "Gluconeogenesis in very low birth weight infants receiving total parenteral nutrition," Diabetes, vol. 48, pp. 791-800, 1999/4/1 1999.

[57] E. E. Tyrala, et al., "Glucose metabolism in the infant weighing less than 1100 grams," The Journal of pediatrics, vol. 125, pp. 283-7, Aug 1994.

[58] A. A. Van Kempen, et al., "Adaptation of glucose production and gluconeogenesis to diminishing glucose infusion in preterm infants at varying gestational ages," Pediatric research, vol. 53, pp. 628-34, Apr 2003.

[59] S. C. Kalhan, et al., "Role of glucose in the regulation of endogenous glucose production in the human newborn," Pediatric research, vol. 20, pp. 49-52, Jan 1986.

[60] R. N. Bergman, et al., "Assessment of insulin sensitivity in vivo," Endocr Rev, vol. 6, pp. 4586, Winter 1985.

[61] L. Chen, "A literature review of intensive insulin therapy and mortality in critically ill patients," Clin Nurse Spec, vol. 24, pp. 80-6, Mar-Apr 2010.

[62] K. Beardsall, et al., "Early Insulin Therapy in Very-Low-Birth-Weight Infants," N Engl J Med, vol. 359, pp. 1873-1884, October 30, 20082008. 
[63] M. J. Schultz, et al., "The new Surviving Sepsis Campaign recommendations on glucose control should be reconsidered," Intensive Care Med, vol. 34, pp. 779-80; author reply 781-2, Apr 2008.

[64] J. C. Preiser and P. Devos, "Clinical experience with tight glucose control by intensive insulin therapy," Crit Care Med, vol. 35, pp. S503-7, Sep 2007.

[65] J. M. Alsweiler, et al., "Survey of the management of neonatal hyperglycemia in Australasia," Journal of Paediatrics and Child Health, vol. 43, pp. 632-635, 2007.

[66] F. Weekers, et al., "Metabolic, endocrine, and immune effects of stress hyperglycemia in a rabbit model of prolonged critical illness," Endocrinology, vol. 144, pp. 5329-38, Dec 2003.

[67] "Maturation of the Glucose Utilization Response to Insulin Occurs before that of Glucose Production in the Preterm Neonate," Pediatric Research Program Issue APS-SPR, vol. 39(4), p. 308, 1996.

[68] J. Lin, et al., "Stochastic Modelling of Insulin Sensitivity Variability in Critical Care," Biomedical Signal Processing \& Control, vol. 1, pp. 229-242, 2006.

[69] J. Lin, et al., "Stochastic modelling of insulin sensitivity and adaptive glycemic control for critical care," Computer Methods and Programs in Biomedicine, vol. 89, pp. 141-152, Feb 2008.

[70] A. Thorell, et al., "Intensive insulin treatment in critically ill trauma patients normalizes glucose by reducing endogenous glucose production," J Clin Endocrinol Metab, vol. 89, pp. 5382-6, Nov 2004.

[71] S. Kalhan, "Metabolism of Glucose and Methods of Investigation in the Fetus and Newborn," in Fetal and Neonatal Physiology, ed, 2003, pp. 449-464.

[72] K. C. McCowen, et al., "Stress-induced hyperglycemia," Crit Care Clin, vol. 17, pp. 107-124, Jan 2001.

[73] L. Jackson, et al., "An inadequate glycemic response to glucagon is linked to insulin resistance in preterm infants?," Arch. Dis. Child. Fetal Neonatal Ed., vol. 88, pp. F62-66, January 1, 2003 2003.

[74] R. Hume, et al., "Glucose homeostasis in the newborn," Early Hum Dev, vol. 81, pp. 95-101, Jan 2005.

[75] W. W. Hay, Jr., et al., "Workshop summary: nutrition of the extremely low birth weight infant," Pediatrics, vol. 104, pp. 1360-8, Dec 1999.

[76] J. Lin, et al., "Stochastic modelling of insulin sensitivity and adaptive glycemic control for critical care," Comput Methods Programs Biomed, Jun 12007.

[77] A. J. Le Compte, et al., "Blood glucose prediction using stochastic modeling in neonatal intensive care," IEEE Trans Biomed Eng, vol. 57, pp. 509-18, Mar 2010. 
Table 1: Patients and/or episodes selected for long-term data collection. Cohort summaries are presented as median [range].

\begin{tabular}{|c|c|c|c|c|c|c|}
\hline Patient & $\begin{array}{l}\text { Gestation age } \\
\text { at birth (weeks) }\end{array}$ & $\begin{array}{l}\text { Age at start } \\
\text { of data } \\
\text { (days) }\end{array}$ & $\begin{array}{c}\text { Weight at } \\
\text { birth (grams) }\end{array}$ & Gender & $\begin{array}{l}\text { Duration of } \\
\text { insulin usage } \\
\text { (days) }\end{array}$ & $\begin{array}{l}\text { Average BG } \\
\text { frequency of } \\
\text { measurements } \\
\text { (per day) }\end{array}$ \\
\hline 1 & 23 & $<1$ & 600 & $\mathrm{~F}$ & 12.7 & 8.6 \\
\hline 2 & 24.4 & 4 & 650 & $\mathrm{~F}$ & 13.9 & 5.5 \\
\hline 3 & 23.7 & $<1$ & 625 & M & 8.8 & 6.0 \\
\hline 4 & 25.4 & $<1$ & 800 & $\mathrm{~F}$ & 12 & 5.5 \\
\hline 5 & 26.6 & 7 & 840 & $\mathrm{~F}$ & 5.9 & 6.2 \\
\hline 6 & 25 & $<1$ & 900 & $\mathrm{~F}$ & 3.9 & 10.9 \\
\hline 7 & 26.3 & 7 & 810 & $M$ & 4.6 & 5.3 \\
\hline 8 & 26.6 & 6 & 825 & $\mathrm{~F}$ & 4.3 & 4.9 \\
\hline 9 & 26.6 & 3 & 915 & M & 3.8 & 6.0 \\
\hline 10 & 27.9 & 3 & 1280 & $\mathrm{~F}$ & 2.7 & 6.9 \\
\hline 11 & 28.1 & $<1$ & 1275 & $\mathrm{~F}$ & 4.9 & 7.5 \\
\hline 12 & 28.6 & $<1$ & 845 & $\mathrm{~F}$ & 1.4 & 10.4 \\
\hline 13 & 27.7 & $<1$ & 860 & $\mathrm{~F}$ & 2.4 & 10.4 \\
\hline 14 & 24.9 & 3 & 735 & M & 7.6 & 7.1 \\
\hline 15 & 26.9 & 2 & 880 & $\mathrm{~F}$ & 8.6 & 6.7 \\
\hline 16 & 29.9 & $<1$ & 865 & $\mathrm{~F}$ & 1.8 & 9.2 \\
\hline 17 & 26.4 & 7 & 990 & M & 1.9 & 5.9 \\
\hline 18 & 26.6 & 3 & 920 & $\mathrm{M}$ & 3.8 & 5.5 \\
\hline 19 & 28.6 & 4 & 930 & $M$ & 1.8 & 11.4 \\
\hline 20 & 26.6 & 4 & 860 & F & 4.7 & 6.0 \\
\hline $21^{\mathrm{a}}$ & 26.6 & 20 & 860 & F & 5.8 & 8.0 \\
\hline 22 & 25.4 & 2 & 800 & $\mathrm{~F}$ & 11.8 & 10.0 \\
\hline $23^{b}$ & 25.4 & 22 & 800 & & 12.7 & 9.2 \\
\hline $24^{\mathrm{b}}$ & 25.4 & 41 & 800 & $F$ & 5.9 & 8.0 \\
\hline $25^{\mathrm{b}}$ & 25.4 & 56 & 800 & & 1.3 & 7.1 \\
\hline Cohort & $\begin{array}{c}26.6 \\
{[23.0-28.9]}\end{array}$ & $\begin{array}{c}3.0 \\
{[<1-56]}\end{array}$ & $\begin{array}{c}845 \\
{[600-1,280]}\end{array}$ & & $\begin{array}{c}4.7 \\
{[1.3-13.9]}\end{array}$ & $\begin{array}{c}7.0 \\
{[4.9-11.4]}\end{array}$ \\
\hline
\end{tabular}


Table 2: Constant model parameter values

\begin{tabular}{ccc}
\hline Parameter & Value & References \\
$k$ & $0.0086 \mathrm{~min}^{-1}$ & {$[33]$} \\
$n$ & $0.90 \mathrm{~min}^{-1}$ & {$[18,19,46,53]$} \\
$\alpha_{I}$ & $1.70 \times 10^{-3} \mathrm{~L} / \mathrm{mU}$ & {$[33]$} \\
$\alpha_{G}$ & $0 \mathrm{~L} / \mathrm{mU}$ & {$[46]$} \\
$C N S$ & $0.088 \mathrm{mmol} / \mathrm{kg} / \mathrm{min}$ & {$[48]$} \\
$m_{\text {brain }}$ & $0.14 * \mathrm{~m}_{\text {body }} \mathrm{kg}$ & {$[49]$} \\
$I_{B}$ & $10 \mathrm{mU} / \mathrm{L} / \mathrm{min}$ & {$[46,53]$} \\
$k_{I}$ & $0.1 \mathrm{~L} /(\mathrm{mU} \mathrm{min})$ & {$[44,45]$} \\
$V_{I, f r a c}$ & $0.045 \mathrm{~L} / \mathrm{kg}$ & {$[39-42]$} \\
$V_{G, \text { frac }}$ & ECF proportion ${ }^{\mathrm{a}} \mathrm{L} / \mathrm{kg}$ & {$[20,53,55-59]$,} \\
$P_{E N D}$ & $0.02838 \mathrm{mmol} / \mathrm{kg} / \mathrm{min}$ & From sensitivity \\
$p_{G}$ & $0.003 \mathrm{~min}^{-1}$ & study \\
\end{tabular}

${ }^{a}$ ECF proportion derived from Figure 1 
Table 3: Quality of fit per-patient compared to model inputs. $\mathrm{BG}_{\text {geo,mean }}$ and $\mathrm{BG}_{\mathrm{geo}, \mathrm{SD}}$ represent the lognormal geometric mean and standard deviation respectively.

\begin{tabular}{|c|c|c|c|c|c|c|}
\hline \multirow[b]{2}{*}{ Patient } & \multirow{2}{*}{$\begin{array}{l}\text { Median } \\
\text { fit error }\end{array}$} & \multirow{2}{*}{$\begin{array}{c}\mathrm{BG}_{\text {geo, mean }} \\
{[\mathrm{mmol} / \mathrm{L}]}\end{array}$} & \multirow{2}{*}{$\begin{array}{l}\mathrm{BG}_{\text {geo, }} \mathrm{SD} \\
{[\mathrm{mmol} / \mathrm{L}]}\end{array}$} & \multirow{2}{*}{$\begin{array}{l}\text { Median } \\
\text { insulin } \\
{[\mathrm{U} / \mathrm{kg} / \mathrm{hr}]}\end{array}$} & \multicolumn{2}{|c|}{ Median dextrose } \\
\hline & & & & & [mg/kg/min] & [kcal/kg/day] \\
\hline 1 & $2.6 \%$ & 6.8 & 1.70 & 0.091 & 8.9 & 51.2 \\
\hline 2 & $2.0 \%$ & 7.8 & 1.26 & 0.053 & 10.4 & 59.7 \\
\hline 3 & $2.7 \%$ & 7.5 & 1.38 & 0.050 & 9.0 & 51.6 \\
\hline 4 & $1.5 \%$ & 7.6 & 1.32 & 0.026 & 8.2 & 47.5 \\
\hline 5 & $3.0 \%$ & 8.0 & 1.37 & 0.037 & 10.5 & 60.6 \\
\hline 6 & $3.6 \%$ & 7.3 & 1.44 & 0.037 & 4.9 & 28.1 \\
\hline 7 & $2.1 \%$ & 7.9 & 1.28 & 0.092 & 11.8 & 68.0 \\
\hline 8 & $1.6 \%$ & 7.7 & 1.28 & 0.031 & 10.7 & 61.4 \\
\hline 9 & $1.0 \%$ & 8.5 & 1.23 & 0.026 & 9.7 & 56.1 \\
\hline 10 & $2.6 \%$ & 6.9 & 1.40 & 0.025 & 9.9 & 56.9 \\
\hline 11 & $1.3 \%$ & 9.1 & 1.57 & 0.047 & 5.9 & 34.2 \\
\hline 12 & $0.6 \%$ & 6.2 & 1.86 & 0.047 & 5.3 & 30.3 \\
\hline 13 & $2.1 \%$ & 5.6 & 1.77 & 0.025 & 4.0 & 23.1 \\
\hline 14 & $1.3 \%$ & 8.9 & 1.28 & 0.029 & 10.0 & 57.7 \\
\hline 15 & $2.1 \%$ & 7.9 & 1.37 & 0.053 & 9.2 & 52.8 \\
\hline 16 & $1.8 \%$ & 8.2 & 1.38 & 0.040 & 5.0 & 28.6 \\
\hline 17 & $4.5 \%$ & 6.3 & 1.46 & 0.059 & 11.4 & 65.4 \\
\hline 18 & $1.5 \%$ & 7.7 & 1.22 & 0.025 & 8.3 & 47.5 \\
\hline 19 & $2.4 \%$ & 8.3 & 1.45 & 0.050 & 11.3 & 65.2 \\
\hline 20 & $2.3 \%$ & 8.4 & 1.30 & 0.030 & 14.1 & 81.4 \\
\hline 21 & $2.2 \%$ & 9.3 & 1.46 & 0.022 & 10.3 & 59.5 \\
\hline 22 & $2.2 \%$ & 7.5 & 1.32 & 0.027 & 8.5 & 49.2 \\
\hline 23 & $6.2 \%$ & 8.2 & 1.54 & 0.077 & 8.8 & 50.6 \\
\hline 24 & $3.1 \%$ & 8.0 & 1.57 & 0.017 & 10.0 & 57.6 \\
\hline 25 & $0.6 \%$ & 9.6 & 1.31 & 0.030 & 9.6 & 55.1 \\
\hline
\end{tabular}


Table 4: Median prediction errors compared to linearly interpolated BG value.

\begin{tabular}{c|c|c}
\multirow{2}{*}{ Prediction interval } & \multicolumn{2}{|c}{ Cohort prediction error } \\
\cline { 2 - 3 } & Median & IQR \\
\hline 1 hour & $5.2 \%$ & {$[2.5 \%-10.3 \%]$} \\
2 hours & $9.4 \%$ & {$[4.5 \%-18.4 \%]$} \\
3 hours & $11.9 \%$ & {$[5.1 \%-23.7 \%]$} \\
4 hours & $13.6 \%$ & {$[6.3 \%-27.6 \%]$} \\
\hline
\end{tabular}

